\title{
Identification of anaplastic lymphoma kinase fusions in clear cell renal cell carcinoma
}

\author{
WEI CHEN $^{1^{*}}$, WEI LI ${ }^{2 *}$, BING BAI $^{3 *}$ and HUAFENG WEI ${ }^{4}$ \\ ${ }^{1}$ Department of Urology, The First Affiliated Hospital of Jiaxing University, Jiaxing, Zhejiang 314001; \\ ${ }^{2}$ Department of Geriatric Neurology, Nanjing Medical University Affiliated to Nanjing Brain Hospital, Nanjing, \\ Jiangsu 210029; ${ }^{3}$ Department of Ultrasonography, Zhejiang Xin'an International Hospital, Jiaxing, Zhejiang 314031; \\ ${ }^{4}$ Cancer Center Laboratory, General Hospital of Chinese PLA, PLA Postgraduate School of Medicine, \\ Beijing 100853, P.R. China
}

Received July 29, 2019; Accepted December 18, 2019

DOI: $10.3892 /$ or.2020.7462

\begin{abstract}
As one of the most common types of renal cancer, clear cell renal cell carcinoma (ccRCC) in advanced stages constitutes a continued major challenge for uro-oncologists, as the identification of novel driver mutations and the development of novel targeted therapies against them remain an unmet need. Aberrations in anaplastic lymphoma kinase (ALK), a rational therapeutic target, as verified in lung cancer with ALK rearrangement, have been implicated in the pathogenesis of multiple human cancers. In the present study, we screened ALK expression in 87 pathologically defined ccRCCs via immunohistochemistry (IHC) using a newly developed rabbit anti-human ALK monoclonal antibody (clone D5F3). Four patients tested positive for ALK expression, as confirmed by IHC. Among them, 2 patients were further confirmed with fluorescence in situ hybridization (FISH) assay with the use of the Vysis LSI ALK dual color break-apart probe. Furthermore, we detected the existence of the echinoderm microtubule-associated protein-like 4/anaplastic lymphoma kinase (EML4-ALK) (E13:A20, variant 1) fusion gene in tumors from these two patients by using rapid amplification of cDNA ends (RACE)-coupled PCR sequencing and RT-PCR. Notably, we first showed that enforced EML4-ALK expression could significantly promote in vitro proliferation, clonogenic colony formation and apoptosis resistance in HK2 immortalized normal renal tubal epithelial cells and their
\end{abstract}

Correspondence to: Dr Huafeng Wei, Cancer Center Laboratory, General Hospital of Chinese PLA, PLA Postgraduate School of Medicine, 28 Fuxing Road, Beijing 100853, P.R. China

E-mail: foxp3_smmu@163.com

*Contributed equally

Key words: ccRCC, clear cell renal cell carcinoma, ALK rearrangement, EML4-ALK, fluorescence in situ hybridization, crizotinib in vivo outgrowth when injected into immunocompromised nude mice. Importantly, this pro-tumorigenic effect was completely abolished by the ALK-specific inhibitor crizotinib, indicating the potential effectiveness of ALK-specific inhibitors in treating ALK-rearranged ccRCC patients. Our data revealed that ALK fusions exist in adult ccRCC, providing a rationale for $\mathrm{ALK}$ inhibitor therapy in selected patients with ccRCC.

\section{Introduction}

As one of the major cancers, renal cancer has a high incidence and mortality rate of approximately 273,518 and 116,368 worldwide, 32,508 and 10,675 in China, and 65,150 and 13,680 in the US, respectively (1). Renal cell carcinoma (RCC) accounts for $90 \%$ of all renal tumors, of which $75 \%$ are clear cell RCC (ccRCC) and $25 \%$ are non-clear cell carcinomas comprising papillary RCC, chromophobe RCC and oncocytoma RCC (2). Although the 5-year survival rate of local RCC patients is as high as 65 to $93 \%$ and as high as 47 to $77 \%$ in stage 1 and stage 2 patients, respectively, approximately $25-30 \%$ of patients with advanced disease have a poor prognosis (i.e., 5-year survival rates ranging from 34 to $80 \%$ and from 2 to $20 \%$ in patients with stage 3 and stage 4 , respectively) $(3,4)$. Several molecularly targeted drugs, including sunitinib, sorafenib and temsirolimus, which mainly target the vascular endothelial growth factor (VEGF) and mammalian target of rapamycin (mTOR) signaling pathways aberrantly activated due to a deficiency in the tumor-suppressor gene von Hippel-Lindau (VHL) in most cases of ccRCC, were recently developed to treat advanced renal cancer (5). Although there has been a significant increase in treatment regimens for advanced $\mathrm{RCC}$, a sustained complete response is infrequent (6).

Anaplastic lymphoma kinase (ALK) is a receptor tyrosine kinase that was first discovered as a fusion gene of nucleophosmin (NPM1) in anaplastic large-cell lymphoma (ALCL) (7). Since then, various $A L K$ fusion genes mediated by translocation have been identified in multiple malignancies, including inflammatory myofibroblastic tumor (IMT), non-small cell lung cancer (NSCLC) and ovarian cancer (8-10). In the scope of the kinase domain, activating mutations with 
ALK have also been identified in neuroblastoma (11-14) and anaplastic thyroid cancer (15). In addition, amplification of the $A L K$ gene has been discovered in neuroblastoma, inflammatory breast cancer (16), and esophageal cancer (17). Although these ALKomas appear in various organs, they share activated ALK with the activity of ALK kinase, which is needed for tumor maintenance (18). Therefore, an aberration in ALK could be used therapeutically as an 'Achilles heel' for tumors. Indeed, it has been reported that there is significant clinical efficacy with ALK inhibitors for NSCLC, ALCL and IMT with ALK fusions (19-22), including crizotinib and ceritinib, ALK-targeting small-molecule tyrosine kinase inhibitors (TKIs). The above have already been approved by the FDA and can be useful for treating NSCLCs positive for ALK rearrangement $(23,24)$. The above findings illustrate that an ALK fusion associated with an oncogene would be one of the most hopeful targets in cancer therapy.

Regarding renal cancer, the $A L K$ fusion gene has also been found in renal medullary carcinoma (RMC) with sickle cell traits and RCC of the unclassified and papillary subtypes (4,25-30); specifically, the VCL-ALK fusion gene was found in 3 patients with RMC. RMC mostly affects young individuals and is associated with poor outcomes, but the finding of $V C L-A L K$ has enhanced the possibility of an effective treatment for patients with an ALK inhibitor. Moreover, the TPM3-ALK or EML4-ALK (E2; A20 variant 5a) fusion has also been detected in a single case each of RCC (4).

Despite sporadic reports in renal cancer, the presence of an ALK fusion has not been found in ccRCC. Herein, we screened 87 patients with ccRCC by immunohistochemistry using a newly developed highly sensitive anti-ALK antibody and detected 4 patients positive for the ALK protein, among which 2 patients were further confirmed as having the EML4-ALK fusion gene by RT-PCR and FISH.

\section{Materials and methods}

Tissues. We examined 24 and 63 renal tumor tissues from ccRCC patients who had undergone surgery at the General Hospital of the Chinese PLA, Beijing between April 2008 and July 2010 and Changhai Hospital affiliated with the Second Military Medical University, Shanghai between May 2008 and December 2010, respectively. The demographic information of the patients is documented in Table I. Surgically removed tumor specimens were fixed in $10 \%$ neutralized formalin and embedded in paraffin for routine histopathological examination. For the 4 cases positive for anti-ALK immunohistochemistry, total RNA was extracted from the corresponding snap-frozen specimen, and purified using RNeasy Mini kit (Qiagen, China). Our study was approval by the Ethics Committee of the General Hospital of the Chinese PLA and Changhai Hospital affiliated to the Second Military Medical University. Frozen normal adult human renal tissues were purchased from Outdo Biotech Co., Ltd., the use of which did not require Institutional Review Board (IRB) approval.

Cell culture. The human renal proximal tubal cell (PTC) line HK2 was obtained from the Cell Collection of the Chinese Academy of Medical Sciences (Beijing, China). The H2228 lung cancer cell line with confirmed EML4-ALK rearrangement (9) was purchased from ATCC. Cells were cultured in complete DMEM (Invitrogen; Thermo Fisher Scientific, Inc.) supplemented with $10 \%$ foetal bovine serum (FBS) and $1 \%$ penicillin/streptomycin/amphotericin B. The culture was carried out in a humidified $5 \% \mathrm{CO}_{2}$ environment at $37^{\circ} \mathrm{C}$. The cells were washed with phosphate-buffered saline (PBS), detached with $0.25 \%$ trypsin $/ 0.2 \%$ EDTA, and plated at $30,000-40,000$ cells $/ \mathrm{cm}^{2}$ when the cells reached $70-80 \%$ confluence. The culture medium was changed one time every 2 days.

Immunohistochemistry. Immunohistochemical staining was conducted on formalin-fixed, paraffin-embedded (FFPE) tissue sections (4- $\mu \mathrm{m}$ thick) as previously described (31). Briefly, the slides were heated for $3 \mathrm{~min}$ in a pressure cooker $\left(100^{\circ} \mathrm{C}\right)$ for antigen retrieval at a concentration of $0.01 \mathrm{~mol} / 1$ Tris-EDTA buffer at pH 8.0 after deparaffinization and rehydration. Under room temperature conditions, all further steps were conducted in a hydrated chamber. Endogenous peroxidase activity was quenched with $3 \%$ hydrogen peroxide for $30 \mathrm{~min}$, and for further study, our precultured slides were incubated with $20 \%$ normal goat serum at the concentration of $50 \mathrm{mmol} / \mathrm{l}$ Tris- $\mathrm{HCl}$ at $\mathrm{pH} \mathrm{7.4.} \mathrm{A} \mathrm{rabbit} \mathrm{monoclonal}$ anti-human ALK antibody from clone D5F3 (cat. \#3633; Cell Signaling Technology) was added at a 1:250 dilution in Dako diluent and incubated for $12 \mathrm{~h}$. Next, the slides were washed with Tris- $\mathrm{HCl}$ and visualized with a horseradish peroxidase-conjugated anti-rabbit EnVision+ Kit (Dako). The sections were then counterstained by using haematoxylin and mounted. ALK-rearranged NSCLC patients served as positive controls and were contained in each staining batch. For the blank control, the same incubation steps were performed, except IgG serum was substituted for the primary antibody (ALK). For CAIX, ABC, CD10, c-Kit, p53, p16, and Ki-67 staining, primary antibodies were anti-ABC clone $\mathrm{W} 6 / 32$ (cat. no. M073601-2; Dako), anti-p53 DO-7 (cat. no. IS61630-2; Dako), anti-CD10 clone 56C6 (cat. no. IS64830-2; Dako), anti-Ki67 clone MIB-1 (cat. no. IS62630-2; Dako) and rabbit polyclonal antibody against human c-Kit (cat. no. A450229-2; Dako), and anti-CAIX clone 2D3 (cat. no. ab107257; Abcam), and anti-p16 clone EPR1473 (cat. no. ab108349; Abcam). Deparaffined sections were incubated with primary antibodies at a 1:20 dilution for $\mathrm{ABC}$ and $\mathrm{p} 53,1: 100$ dilution for $\mathrm{CD} 10$ and p16, and 1:80 dilution for c-Kit, CAIX and $\mathrm{Ki}-67$, at $4^{\circ} \mathrm{C}$, overnight. After washing in PBS, the sections were visualized and counterstained as described above. PBS substituted for the primary antibody was used as the negative control.

ALK fusion analysis by rapid amplification of cDNA ends (RACE)-coupled PCR sequencing. We adopted the method of RACE-coupled PCR sequencing to detect the fusion partner of ALK as described previously (32). Briefly, an ALK gene-specific primer was applied to reverse transcribe RNAs into cDNAs. Next, cDNAs were purified and further subjected to polycytidine (poly-C) tailing. Two PCRs were carried out to amplify the target cDNA fragments spanning exon 20 of ALK and its upstream sequence, which may include the transcript sequence of any gene fused to ALK. Then, the resultant PCR products were purified and sequenced using the M13 
Table I. Clinicopathological characteristics of the patients with ccRCC in the present study.

\begin{tabular}{lc}
\hline Variable/group & Data \\
\hline Age (years) & \\
Median & 56 \\
Range & $28-79$ \\
Sex, n (\%) & \\
Male & $62(71.2)$ \\
Female & $25(28.8)$ \\
Stage ${ }^{\mathrm{a}}, \mathrm{n}(\%)$ & \\
I & $50(57.5)$ \\
II & $22(25.3)$ \\
III & $10(11.5)$ \\
IV & $5(5.7)$ \\
Total & $87(100)$ \\
\hline
\end{tabular}

astage was determined according to the 7th edition of the AJCC TNM Cancer Staging Manual. ccRCC, clear cell renal cell carcinoma.

sequencing primer. Target sequences of interest were aligned with the ALK reference sequence (NM_004304.3) to confirm its fusion with another gene.

$R T$-PCR and sequencing. Reverse transcription polymerase chain reaction (RT-PCR) was carried out with total RNA extracted from frozen tumor tissues by the use of TRIzol reagent (Invitrogen; Thermo Fisher Scientific, Inc.). Reverse transcription was conducted with a RevertAid First Strand cDNA Synthesis Kit (Thermo Fisher Scientific, Inc.). Simultaneously, PCR was carried out with primers specific to EML4-ALK (EML4 exon 2-ALK exon 20) and GAPDH, as follows: Forward, 5'-TGTGCTCTGAACAGGACG AACT-3' and reverse, 5'-GCCTCCACTGGTGACAAA CTC-3' (1,399 bp for EML4-ALK variant 1 and 575 bp for EML4-ALK variant 3); and forward, 5'-CCATGTTCGTCA TGGGTGTGAACCA-3' and reverse, 5'-GCCAGTAGAGGC AGGGATGATGTTC-3' (250 bp). Direct sequencing was carried out on the PCR products by the use of forward and reverse PCR primers.

Fluorescence in situ hybridization. Fluorescence in situ hybridization (FISH) analysis was conducted on $4-\mu \mathrm{m}$ thick FFPE tissue sections of 2 patients with ccRCC positive for ALK expression as determined by IHC using the Vysis LSI ALK dual colour break-apart probe (Abbott Molecular). Next, hybridized slides were stained with 4',6-diamidino-2-phenylindole and measured on an FV1000 confocal laser scanning microscope at x400 magnification (Olympus, Tokyo, Japan).

EML4-ALK expression in HK2 cells. The retroviral vector pMXS expressing the bicistronic EML4-ALK fusion gene and mouse CD8 was the gift of Dr Hiroyuki Mano of Jichi Medical University (9). The retrovirus was prepared by transiently cotransfecting three plasmids (pMXS or pMXS-EML4-ALK, psPAX2, and pMD2.G), as previously described (31). The resultant virus was used to infect HK2 cells, and then cells stably expressing the control or the EML4-ALK fusion gene were obtained by flow cytometry by using a mouse CD8 marker. EML4-ALK expression was confirmed by western blotting with an ALK-specific antibody (D5F3).

Colony formation assays, proliferation assays and Annexin V/PI staining. All assays were performed as previously described (31). Briefly, cells were plated in triplicate wells of 6 -well dishes at a low density ( 150 cells/well) and cultured under normal conditions without perturbation. After 10 days, the colonies were washed with PBS and stained with crystal violet $(0.5 \% \mathrm{w} / \mathrm{v}$ in $25 \%$ methanol). Stained plates were rinsed in $\mathrm{ddH}_{2} \mathrm{O}$ and allowed to dry at room temperature. The plates were photographed, and colonies were counted using Image J 1.8.0 software (NIH; National Institutes of Health, Bethesda, MD, USA). For the proliferation assay, cells $\left(3 \times 10^{3}\right.$ per well) were cultured in 6 -well plates in medium containing complete supplements. Cell proliferation was detected on a hemocytometer by cell counting every 3 days. For Annexin V/PI staining, cells $\left(1 \times 10^{5}\right.$ per well $)$ were cultured in 6-well plates with or without serum for $24 \mathrm{~h}$ and subjected to Annexin V/PI staining on the basis of the manufacturer's instructions (Vazyme, Nanjing, China) and immediately analyzed by flow cytometry.

Xenograft model in nude mice. Animal care and protocols were reviewed and approved by the Institutional Animal Care and Use Committee of the General Hospital of the Chinese PLA. Forty 5-6 week-old female BALB/c nude mice with initial weight of $\sim 20 \mathrm{~g}$ were obtained from Weitonglihua Biotechnology (Beijing, China) and maintained on a $12-\mathrm{h}$ light-dark cycle in a temperature-controlled high barrier facility with free access to food and water and treated under specific pathogen-free conditions at the Animal Centre of the General Hospital of the Chinese PLA. The xenograft model was established by subcutaneously injecting HK2 cells stably expressing control or EML4-ALK $\left(3 \times 10^{6}\right.$ cells per mouse) into the right flanks of mice. When the xenograft reached $100-150 \mathrm{~mm}^{3}$ (control HK2) or $250-350 \mathrm{~mm}^{3}$ (EML4-ALK HK2), animals were randomly separated into two groups (5 mice per group) and intraperitoneally injected with vehicle (DMSO; from Sigma-Aldrich; Merck KGaA) or crizotinib $(250 \mathrm{mg} / \mathrm{kg}$ reconstituted in DMSO; from Selleck) twice weekly for 2 weeks. Mice were weekly monitored for tumou growth by using a calliper for 30 days, and then euthanized by cervical dislocation with tumor harvested for imaging when they seemed moribund or their tumors reached $15 \mathrm{~mm}$ in diameter. Tumor volume (V) was calculated according to the following formula: $\mathrm{V}\left(\mathrm{mm}^{3}\right)=$ length $\mathrm{x}$ width ${ }^{2} / 2$.

Statistical analysis. Statistical analyses were carried out with GraphPad Prism (version 5.04, GraphPad Software, Inc.). The results are presented as the mean $\pm \mathrm{SD}$ obtained from at least three independent experiments. Differences in groups were determined by two-way ANOVA with multiple comparisons and Bonferroni correction or two-tailed paired Student's t-test with $\mathrm{P}<0.05$ considered to indicate a statistically significant result. 

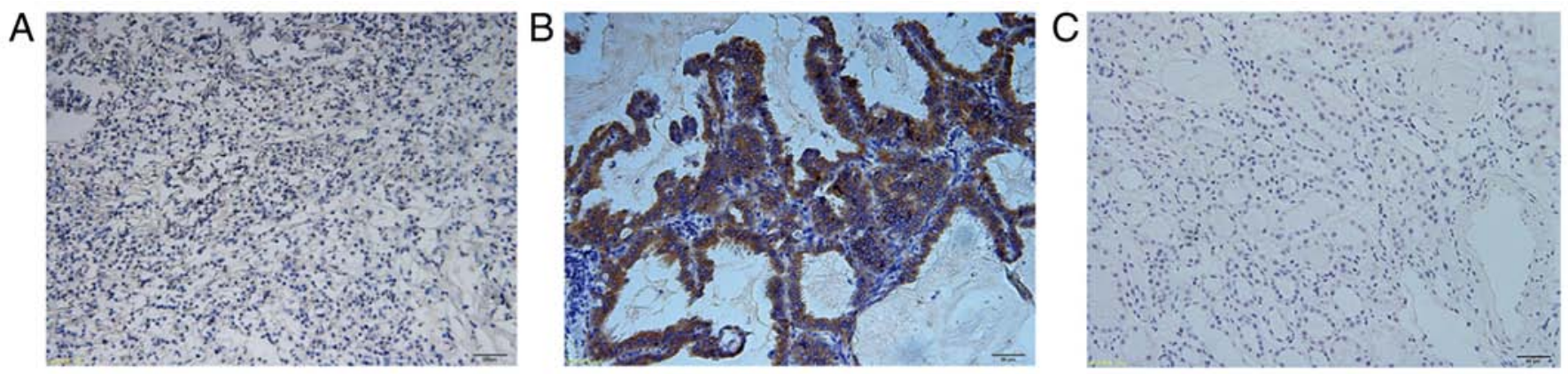

Figure 1. Photomicrographs of ALK-germline and ALK-rearranged lung adenocarcinomas and normal adult human renal tissues stained with the D5F3 antibody. (A) ALK-germline sample. Scale bar, $50 \mu \mathrm{m}$. (B) ALK-rearranged sample. Scale bar, $50 \mu \mathrm{m}$. (C) Normal adult human renal tissues. Scale bar, $100 \mu \mathrm{m}$. ALK. anaplastic lymphoma kinase.
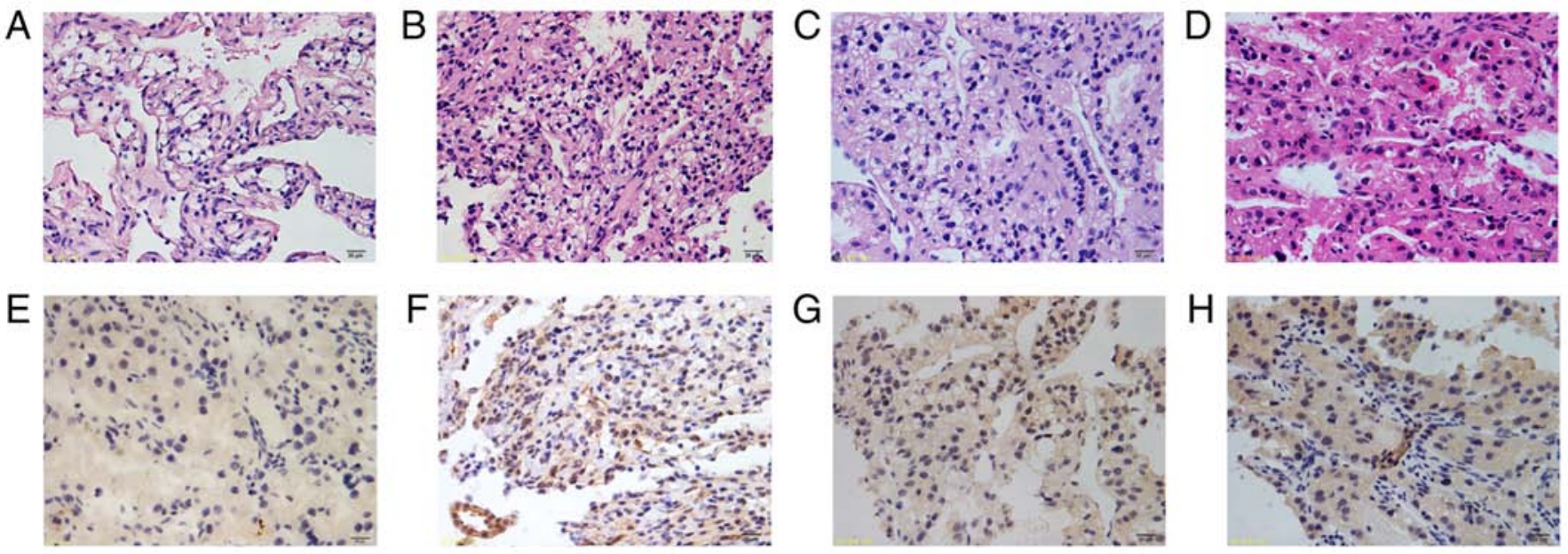

Figure 2. Photomicrographs of ccRCC tissues stained with the D5F3 antibody showing positive expression. Sample 416 (A and E) sample 413 (B and F) sample 405 (C and G) and sample 384 (D and H). (A-D and E-H) Haematoxylin and eosin (H\&E) staining and ALK staining, respectively. Scale bar, $20 \mu$ m. ccRCC, clear cell renal cell carcinoma; ALK, anaplastic lymphoma kinase.

\section{Results}

Detection of ALK protein expression in ccRCC tumor samples by $I H C$. We screened ALK protein expression in the FFPE specimens of 87 pathologically defined ccRCCs by IHC with a highly sensitive rabbit anti-human ALK monoclonal antibody, D5F3, which was reported to possess $100 \%$ sensitivity and 99\% specificity in the measurement of ALK protein expression from NSCLC tumor samples in a previous study (33). We first optimized standard immunohistochemical staining using known ALK-rearranged and ALK-germline NSCLC tumor samples, and each case was confirmed by FISH. As shown in Fig. 1A and B, the D5F3 antibody displayed robust tumor-specific staining in ALK-rearranged tumors and essentially no tissue staining in ALK-germline tumors using a wide scope of antibody dilutions and antigen retrieval methods (data not shown). There is also a lack of staining in normal adult human renal tissue which is known to not express ALK (18), further validating the staining specificity of this antibody (Fig. 1C). Then, ccRCC tissue sections were immunostained for ALK expression using the same protocol, and 4 positive samples with middle-level staining were detected (sample 416, Fig. 2A and E; sample 413, Fig. 2B and F; sample 405, Fig. 2C and G; sample 384 Fig. 2D and H).
Identification of the 5' ALK fusion partner and EML4-ALK expression. We performed 5'-RACE assays to determine whether samples with ALK expression detected by IHC expressed the wild-type or fusion ALK gene. The first round of 5'-RACE did not yield products that could be visualized on the gel; however, an approximate 1,800-bp band was obvious in the nested reaction of samples 405 and 413 . The nested reaction included a portion of ALK cDNA (exon 20) immediately preceded with a non-ALK sequence, which was confirmed by sequencing analysis of the purified and cloned products. Furthermore, BLASTN analysis indicated that the non-ALK sequence reached $100 \%$ and was identical to a portion of EML4 mRNA (NCBI reference number: NM_3373) that included exons 1 through 13 of the gene. Confirmatory RT-PCR conducted on the independently synthesized tumor cDNA with EML4 exon 2 forward and ALK exon 20 reverse primers produced a predicted 1399-bp product (Fig. 3A), the sequencing of which confirmed the breakpoint (Fig. 3B). This EML4-ALK product is named variant 1 (E13; A20). To determine the genomic rearrangement, we performed ALK-split FISH assays. As shown in Fig. 3C, FISH demonstrated that 30 and $24 \%$ of scored nuclei in samples 405 and 413, respectively, displayed split ALK signals, in accordance with a rearrangement. We did not obtain appreciable bands 
A

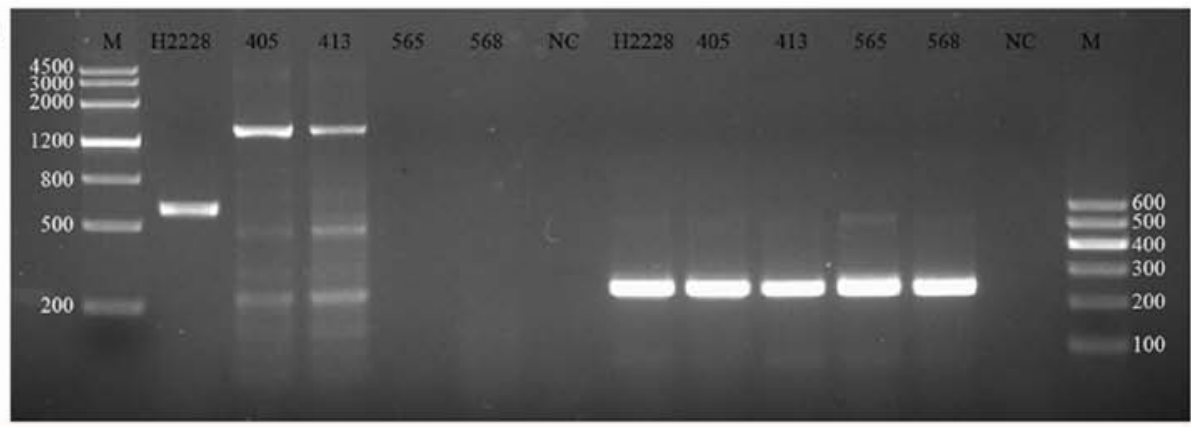

B
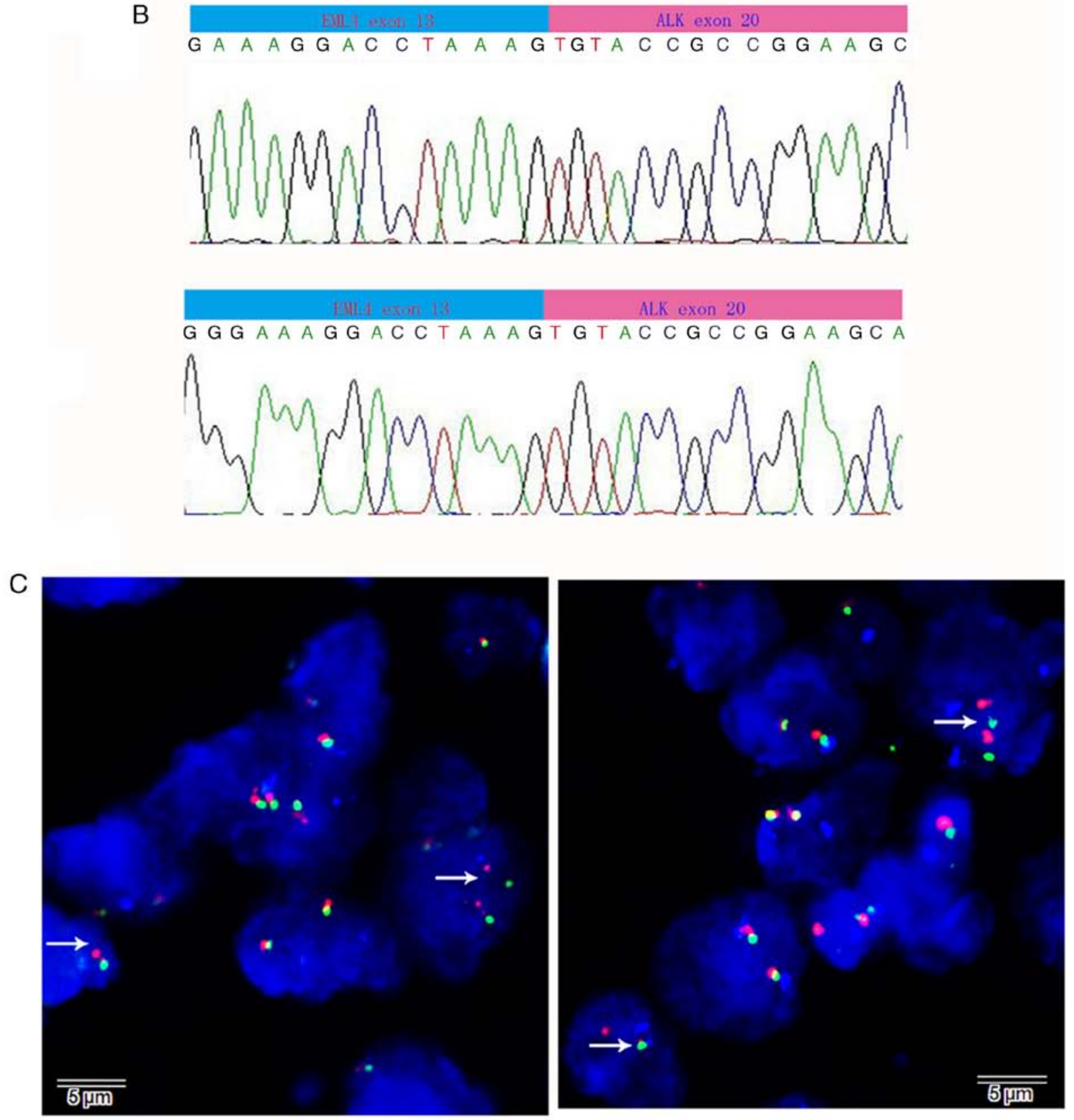

Figure 3. Identification of the 5' ALK fusion partner and EML4-ALK expression. (A) Electrophoresis of products from the RT-PCR analysis of tumor cDNA with the EML4 exon 2 forward and ALK exon 20 reverse primers shows an expected 1,399-bp product (column 3, sample 405; column 4, sample 413). In column 2, cDNA from H2228 lung cancer cells containing a known $E M L 4-A L K$ fusion gene (variant 3) was amplified by the same primers, which showed an expected 599-bp product. In columns 5 and 6, samples 565 and 568, with negative ALK expression by IHC, were included as the negative control. In columns 8-12, GAPDH was amplified as a positive control (250 bp), and in columns 3 and 7, a negative control reaction without cDNA was run. In columns 1 and 14, DNA molecular markers are shown. (B) Sequencing results from samples 405 (upper panel) and 413 (lower panel) show the sequence of the $E M L 4-A L K$ fusion breakpoint; the DNA base on EML4 exon 13 (blue) is immediately followed by the DNA base on ALK exon 20 (pink). (C) FISH analysis with the Vysis LSI ALK dual colour break-apart probe confirmed the presence of ALK rearrangement. The white arrow denotes split red-green signals indicative of ALK rearrangement, while touching red-green signals are not indicative of an ALK rearrangement. EML4-ALK, echinoderm microtubule-associated protein-like 4/anaplastic lymphoma kinase; FISH, fluorescence in situ hybridization. 
from samples 416 or 384 by 5'-RACE; additionally, RT-PCR with the primers used to amplify known ALK fusion genes, including EML4-ALK, VCL-ALK, TMP3-ALK, NPM1-ALK, $T F G$-ALK, ATIC-ALK and CLTC-ALK, showed no specific bands (data not shown).

Case report and pathological findings. Samples 405 and 413 were obtained from 38- and 59-year-old male patients, respectively, with ccRCC of Fuhrman grade II diagnosed in March 2012. Patient 405 had a local tumor $4 \mathrm{~cm}$ in diameter on his right kidney, and his tumor tissue presented gland ducts and an acinar arrangement, cuboidal cells, a translucent cytoplasm, a round nucleus, a nucleolus partially visible under low magnification, and blood sinus dilatation and congestion (Fig. 4A). After surgery, he remains in good health, with no evidence of disease at present. Patient 413 presented with a tumor $4.5 \mathrm{~cm}$ in diameter on his left kidney, with a cancer embolus noted in the blood vessels around the tumor that invaded the renal capsule. His tumor tissue showed a group-like arrangement with rounded and polygonal cancer cells, a translucent cytoplasm, a small round nucleus and vaguely visible nucleoli, and rich vascularity in the interstitial space (Fig. 4B). After surgery, he remained in good health until December 2015, when a routine examination showed that he had a new small tumor $0.4 \mathrm{~cm}$ in diameter on the right kidney, and he is now under intensive observation without further treatment. According to IHC, these two neoplasms were positive for ABC, CAIX and CD10 (Fig. 4) and negative for c-KIT, p53 and p16, with a relatively low Ki-67 index (5\% staining; data not shown).

Functional effect of the EML4-ALK fusion gene in renal epithelial cells. To further study the effect of EML4-ALK expression on renal carcinogenesis, we carried out correlative research on the biological effects of EML4-ALK. First, we steadily expressed EML4-ALK by the use of retrovirus transduction in HK2 cells, a deathless human renal proximal tubal epithelial cell line lacking endogenous EML4-ALK expression (Fig. 5A). EML4-ALK expression in HK2 cells enhanced cell colony formation and cell proliferation in vitro (Fig. 3B and C). Furthermore, EML4-ALK expression protected HK2 cells from serum starvation-induced apoptosis (Fig. 5D). In addition, we evaluated the protumorigenic effect of EML4-ALK expression in vivo. As shown in Fig. 5E, EML4-ALK expression promoted the outgrowth of HK2 cell-derived xenografts in nude mice $\left(232 \pm 164.6\right.$ vs. $1,311.2 \pm 1,261.6 \mathrm{~mm}^{3}$ for Control/Vehicle vs EML4-ALK/Vehicle, $\mathrm{P}<0.001)$, consistent with the transforming activity of this fusion gene previously reported (9); more importantly, the inhibition of ALK activity with the small-molecule TKI crizotinib completely abolished the protumor effect of EML4-ALK expression in this context (357.25 \pm 262.5 vs. $440.75 \pm 110.4 \mathrm{~mm}^{3}$ for Control/Crizotinib vs. EML4-ALK/Crizotinib, P>0.05), indicating the therapeutic potential of ALK-targeting TKIs in ccRCC with ALK rearrangement.

\section{Discussion}

An anaplastic lymphoma kinase (ALK) rearrangement is a key molecular event resulting in the emergence of functional $A L K$ fusion genes capable of driving carcinogenesis, and approximately $30 A L K$ fusion genes with different partners have been identified in a plethora of tumor types $(18,34)$. Importantly, the targeted inhibition of $A L K$ fusion genes by small-molecule tyrosine kinase inhibitors (TKIs) has demonstrated an impressive clinical outcome in treating cancer patients with ALK rearrangements confirmed by RT-PCR, immunohistochemistry (IHC) and/or fluorescence in situ hybridization (FISH) $(18,34)$. Thus, the identification of ALK rearrangement events and the elucidation of their functional effects have always been of clinical significance. In the present study, we screened ALK expression in clear cell renal cell carcinoma (ccRCC) patients via routine IHC techniques and found 4 patients with positive ALK staining that were verified to have the EML4-ALK rearrangement by RT-PCR and FISH techniques.

Previous studies screened 1,714 patients with renal cancers, including renal medullary carcinoma (RMC), ccRCC and papillary RCC, and identified 8 ALK rearrangement-positive patients among which two groups were established (4,25-30): three cases of $V C L-A L K$-rearranged paediatric tumors with sickle cell traits and 5 cases of adult tumors with a papillary architecture and an alternative $A L K$ fusion partner (e.g., TPM4 or ELM4). Our identification of the EML4-ALK fusion gene in 2 patients with ccRCC constitutes the third group. Current data from 6 previous studies and ours on $10 A L K$-rearranged tumors among 1,801 representative RCCs with main morphologies, which were observed in different ethnicities of pediatric and adult patients (Table II), demonstrate a very low overall frequency $(0.55 \%)$ of $A L K$ rearrangement in RCC. Currently, no consistent pathological features of all ALK-rearranged RCCs have been revealed except for the presence of a non-specific eosinophilic cytoplasm (35). Smith et al summarized the distinct features of ALK-rearranged RMC patients: sickle-cell traits, an age of approximately 9.3 years, polygonal spindle-shaped cells with obvious cytoplasmic vacuoles, a small Ki-67 index and pristine INI-1 (27). However, no distinct features were noted for patients with ALK-rearranged RCC other than RMC; thus, as mentioned by Debelenko, it appears that tumor histology cannot be used to predict $A L K$ rearrangement and that ALK-rearranged RCC represents an individual pathologic subtype would also be impractical (35).

Although it has been well documented that $A L K$ fusion genes confer transforming activity in multiple cancers (18), including non-small cell lung cancer (NSCLC) and anaplastic large-cell lymphoma (ALCL) $(7,9)$, it has never been determined whether the same is true in the setting of RCC. Using overexpression experiments in the immortalized renal epithelial cell line HK2, we first showed that ELM4-ALK expression significantly promoted the malignant behaviors of otherwise normal cells in vitro, conferring a tumorigenic effect in vivo. More importantly, the ALK-targeted TKI crizotinib nearly completely suppressed the protumour effect of ELM4-ALK in the HK2 xenograft model, preliminarily validating the effectiveness of ALK-targeted therapy in treating ALK-rearranged RCC. However, one limitation of our findings is that we cannot rule out the influence and contribution of the host on the final analysis. Certainly, it would be more clinically relevant if the efficacy of ALK-targeted TKIs is tested in xenografts from tumor tissues of ALK-rearranged RCC patients. 
A

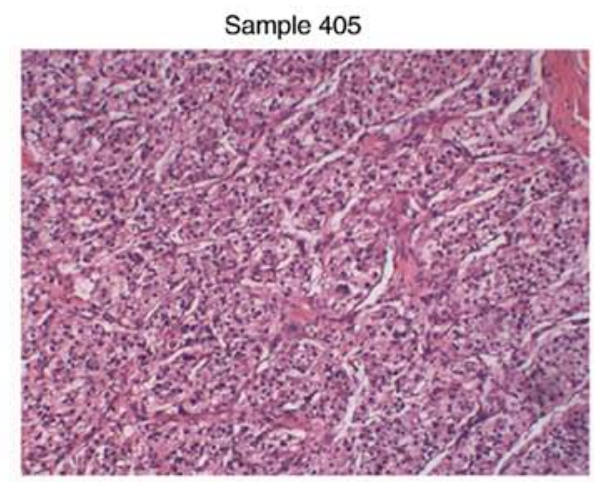

H\&E $(\times 100)$

H\&E (×200)

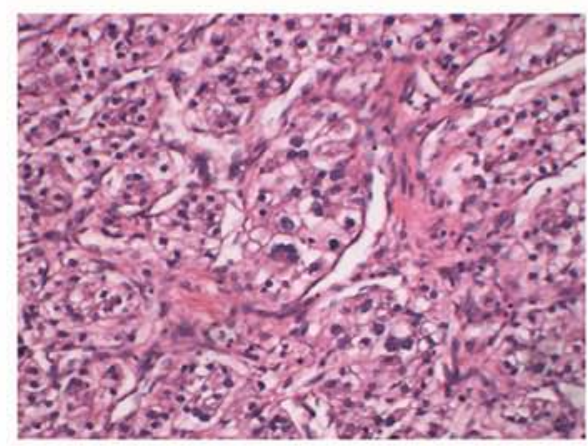

$A B C(\times 200)$

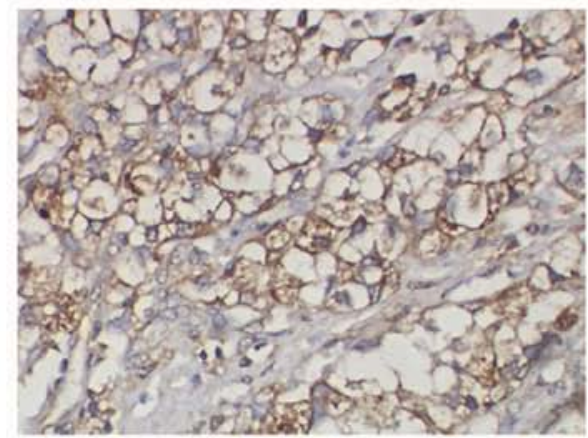

CAIX $(\times 200)$

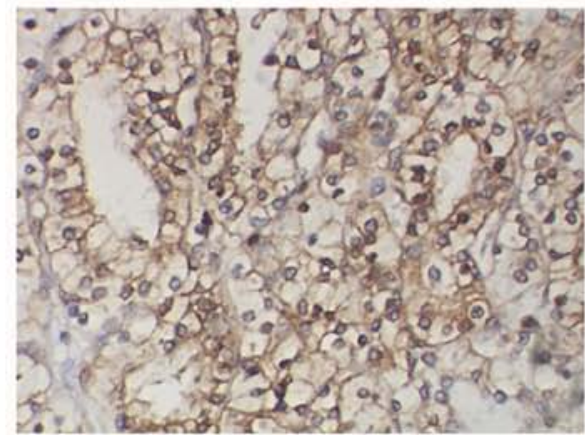

CD10 $(\times 200)$

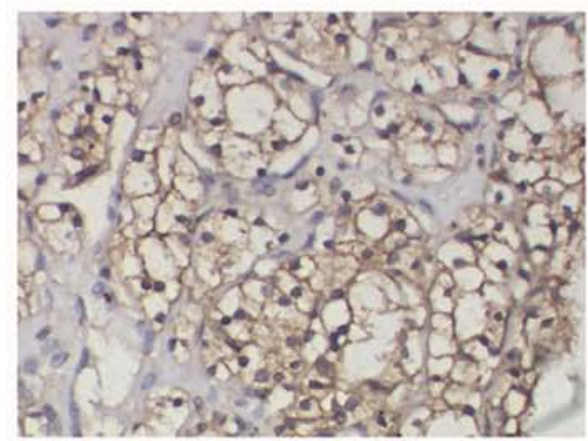

B
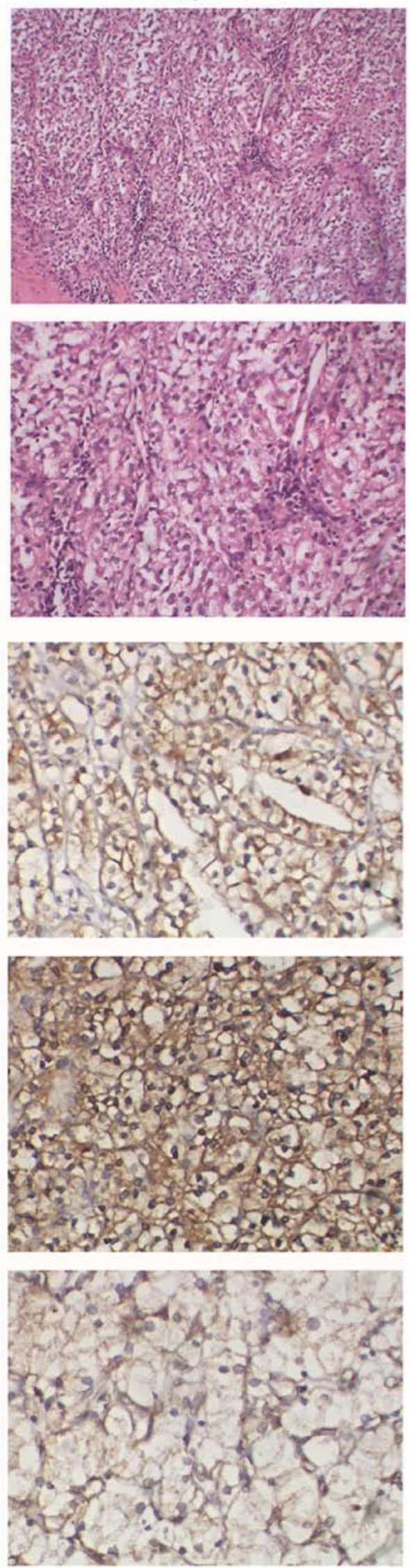

Figure 4. Histological and immunohistochemical analyses of ALK-rearranged cases. (A) Sample 405; this patient's tumor tissue presents gland ducts and an acinar arrangement, cuboidal cells, a translucent cytoplasm, a round nucleus, nucleolus partially visible under low magnification, and blood sinus dilatation and congestion. (B) Sample 413; this patient tumor tissue shows a group-like arrangement with rounded and polygonal cancer cells, a translucent cytoplasm, a small round nucleus and vaguely visible nucleoli, and rich vascularity in the interstitial space. These two neoplasms were positive for carbonic anhydrase 9 (CAIX), HLA class I (ABC) and cluster of differentiation 10 (CD10). ALK, anaplastic lymphoma kinase. 
A

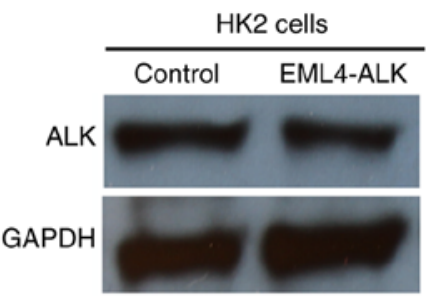

C

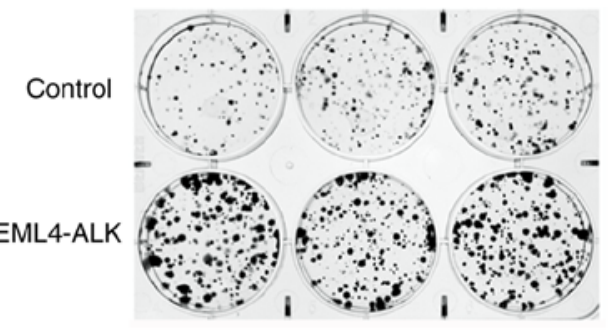

D

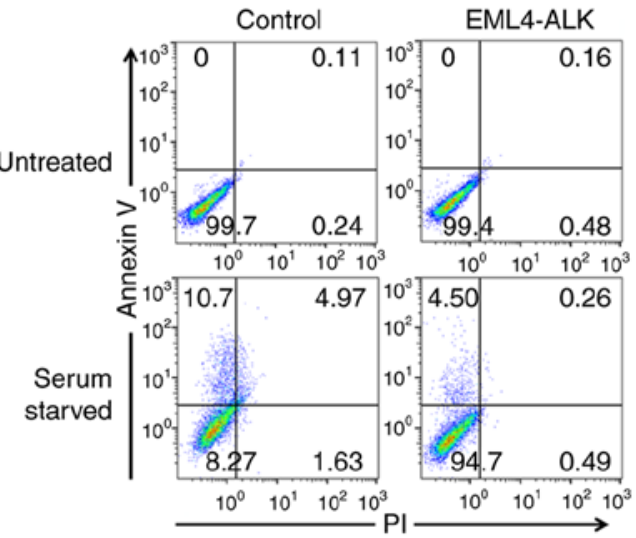

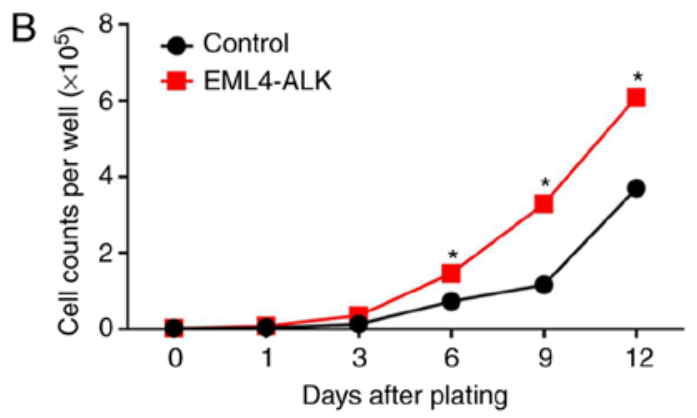
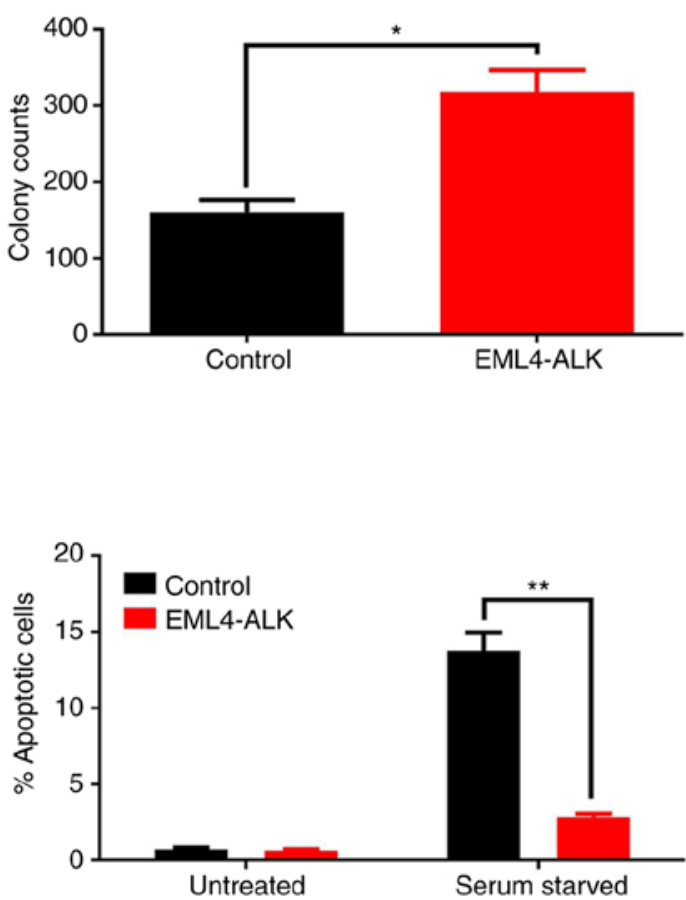

E
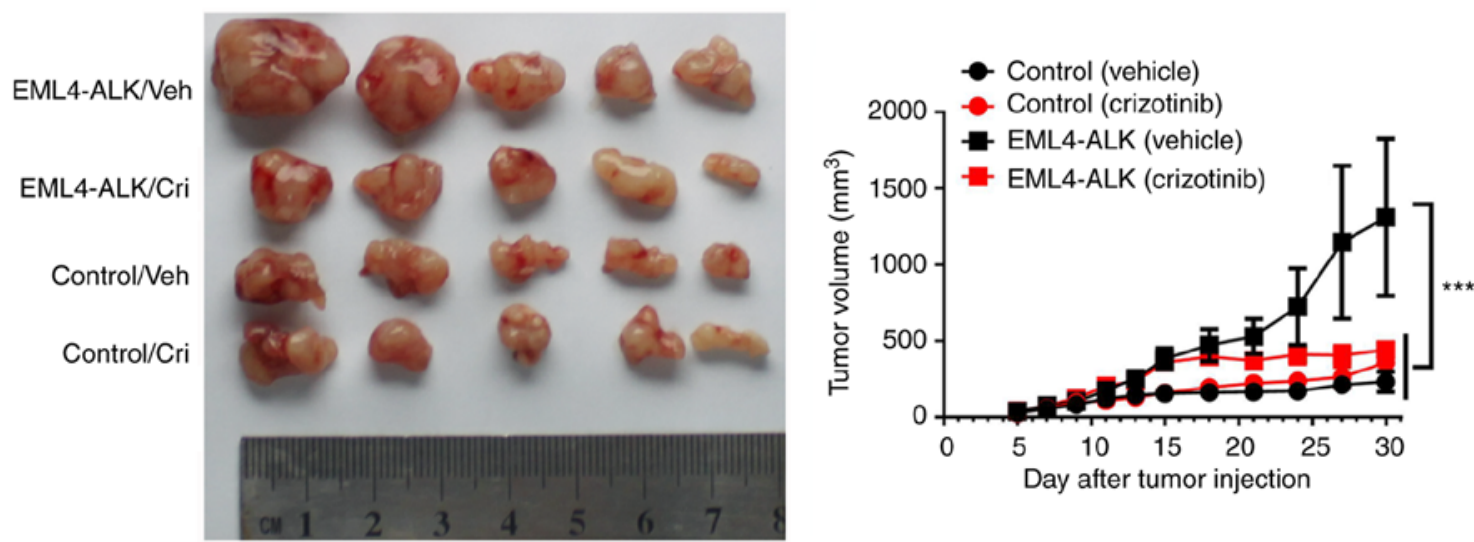

Figure 5. EML4-ALK overexpression has a protumorigenic effect on normal renal epithelial cells. (A) EML4-ALK expression in HK2 cells stably infected with a control or EML4-ALK-carrying retrovirus determined by western blot analysis. In vitro (B) proliferation and (C) clonogenic colony formation assays. HK2 cells stably expressing the control or EML4-ALK retrovirus were plated into 6-well plates and cultured for 10 or 12 days, and cell counts were performed every 3 days (B) and colonies were imaged $(\mathrm{C}$, left) and counted at the end of the experiment (C, right). The data are expressed as the mean \pm SD of triplicate samples. (D) HK2 cells stably expressing the control or EML4-ALK retrovirus were plated into 6-well plates with (untreated) or without serum (serum starved) for $24 \mathrm{~h}$, and cell apoptosis was analyzed by Annexin V/PI staining and flow cytometry. The data are expressed as the mean \pm SD of triplicate samples. (E) Tumors were established by subcutaneously injecting HK2 cells stably expressing the control or EML4-ALK retrovirus into the right flanks of nude mice. When the xenograft reached $100-150 \mathrm{~mm}^{3}$ (control HK2) or 250-350 $\mathrm{mm}^{3}$ (EML4-ALK HK2), animals were randomly divided into two groups and intratumorally injected with vehicle (DMSO) or crizotinib $(250 \mathrm{mg} / \mathrm{kg}$ ) twice weekly for 2 weeks. Tumor formation was monitored, harvested tumors were imaged (E, left), and their volume was calculated (E, right). Each time point represents the mean tumor volume for each group. Data are representative of 2-3 independent experiments. ${ }^{*} \mathrm{P}<0.05,{ }^{* *} \mathrm{P}<0.01,{ }^{* * *} \mathrm{P}<0.001$, paired Student's t-test (for $\mathrm{B}, \mathrm{C}$ and $\mathrm{D}$ ) or two-way ANOVA with multiple comparisons corrected with the Bonferroni method (for E). EML4-ALK, echinoderm microtubule-associated protein-like 4/anaplastic lymphoma kinase. 
In summary, we identified two patients with $A L K$ rearrangement among 87 Chinese ccRCC patients. Furthermore, the $E M L 4-A L K$ (E13: A20 variant 1) fusion gene was revealed in these two patients, whose protumor effect and its effectiveness as a therapeutic target were tested in an animal xenograft model. There is no clinical trials or case report of an ALK inhibitor in treating ALK-rearranged RCC to date; however, ALK-RCC has been recently proposed and incorporated into the recent World Health Organisation Classification of Renal Tumours as a provisional entity (36); as ALK-targeted TKIs have shown impressive therapeutic efficacy in treating patients with ALK rearrangement in NSCLC and other cancers, it is possible that ALK inhibitor therapy will provide great benefit for patients with advanced stage ALK-rearranged ccRCC in the near future.

\section{Acknowledgements}

Not applicable.

\section{Funding}

This work was supported by the National Natural Science Foundation of China (nos. 81372528 and 81672274) and the National High Technology Research and Development Program ('863' Program) of China (no. 2014AA020704).

\section{Availability of data and materials}

The datasets and certain material used and/or analyzed during the present study are available from the corresponding author on reasonable request.

\section{Authors' contributions}

WC and HW conceived the study. WC, WL and BB performed the experiments. WC and HW analyzed the data and prepared the manuscript. All authors read and approved the final manuscript. All authors read and approved the manuscript and agree to be accountable for all aspects of the research in ensuring that the accuracy or integrity of any part of the work are appropriately investigated and resolved.

\section{Ethics approval and consent to participate}

Human tissues were provided by the Department of Pathology, General Hospital of Chinese PLA (Beijing) and Changhai Hospital affiliated to Second Military Medical University (Shanghai) following approval by the Ethics Committee of the General Hospital of Chinese PLA and Changhai Hospital affiliated to the Second Military Medical University.

\section{Patient consent for publication}

Not applicable.

\section{Competing interests}

The authors declare that they have no competing interests. 


\section{References}

1. Ferlay J, Shin HR, Bray F, Forman D, Mathers C and Parkin DM Estimates of worldwide burden of cancer in 2008: GLOBOCAN 2008. Int J Cancer 127: 2893-2917, 2010.

2. Lopez-Beltran A, Carrasco JC, Cheng L, Scarpelli M, Kirkali Z and Montironi R: 2009 update on the classification of renal epithelial tumors in adults. Int J Urol 16: 432-443, 2009.

3. Rini BI, Campbell SC and Rathmell WK: Renal cell carcinoma. Curr Opin Oncol 18: 289-296, 2006.

4. Sugawara E, Togashi Y, Kuroda N, Sakata S, Hatano S, Asaka R, Yuasa T, Yonese J, Kitagawa M, Mano H, et al: Identification of anaplastic lymphoma kinase fusions in renal cancer: Large-scale immunohistochemical screening by the intercalated antibody-enhanced polymer method. Cancer 118: 4427-4436, 2012.

5. Pécuchet N, Fournier LS and Oudard S: New insights into the management of renal cell cancer. Oncology 84: 22-31, 2013.

6. Singer EA, Gupta GN and Srinivasan R: Targeted therapeutic strategies for the management of renal cell carcinoma. Curr Opin Oncol 24: 284-290, 2012.

7. Morris SW, Kirstein MN, Valentine MB, Dittmer KG, Shapiro DN, Saltman DL and Look AT: Fusion of a kinase gene, ALK, to a nucleolar protein gene, NPM, in non-Hodgkin's lymphoma. Science 263: 1281-1284, 1994.

8. Lawrence B, Perez-Atayde A, Hibbard MK, Rubin BP, Dal Cin P, Pinkus JL, Pinkus GS, Xiao S, Yi ES, Fletcher CD and Fletcher JA: TPM3-ALK and TPM4-ALK oncogenes in inflammatory myofibroblastic tumors. Am J Pathol 157: 377-384, 2000

9. Soda M, Choi YL, Enomoto M, Takada S, Yamashita Y, Ishikawa S, Fujiwara S, Watanabe H, Kurashina K, Hatanaka $\mathrm{H}$, et al: Identification of the transforming EML4-ALK fusion gene in non-small-cell lung cancer. Nature 448: 561-566, 2007.

10. Ren H, Tan ZP, Zhu X, Crosby K, Haack H, Ren JM, Beausoleil S Moritz A, Innocenti G, Rush J, et al: Identification of anaplastic lymphoma kinase as a potential therapeutic target in ovarian cancer. Cancer Res 72: 3312-3323, 2012.

11. Mossé YP, Laudenslager M, Longo L, Cole KA, Wood A, Attiyeh EF, Laquaglia MJ, Sennett R, Lynch JE, Perri P, et al: Identification of ALK as a major familial neuroblastoma predisposition gene. Nature 455: 930-935, 2008.

12. Janoueix-Lerosey I, Lequin D, Brugières L, Ribeiro A, de Pontual L, Combaret V, Raynal V, Puisieux A, Schleiermacher G, Pierron G, et al: Somatic and germline activating mutations of the ALK kinase receptor in neuroblastoma. Nature 455: 967-970, 2008.

13. George RE, Sanda T, Hanna M, Fröhling S, Luther W II, Zhang J, Ahn Y, Zhou W, London WB, McGrady P, et al: Activating mutations in ALK provide a therapeutic target in neuroblastoma. Nature 455: 975-978, 2008.

14. Chen Y, Takita J, Choi YL, Kato M, Ohira M, Sanada M, Wang L, Soda M, Kikuchi A, Igarashi T, et al: Oncogenic mutations of ALK kinase in neuroblastoma. Nature 455: 971-974, 2008.

15. Murugan AK and Xing M: Anaplastic thyroid cancers harbor novel oncogenic mutations of the ALK gene. Cancer Res 71 : 4403-4411,2011.

16. Tuma RS: ALK gene amplified in most inflammatory breast cancers. J Natl Cancer Inst 104: 87-88, 2012.

17. Schoppmann SF, Streubel B and Birner P: Amplification but not translocation of anaplastic lymphoma kinase is a frequent event in oesophageal cancer. Eur J Cancer 49: 1876-1881, 2013.

18. Mano H: ALKoma: A cancer subtype with a shared target. Cancer Discov 2: 495-502, 2012

19. Kwak EL, Bang YJ, Camidge DR, Shaw AT, Solomon B, Maki RG, Ou SH, Dezube BJ, Jänne PA, Costa DB, et al: Anaplastic lymphoma kinase inhibition in non-small-cell lung cancer. N Engl J Med 363: 1693-1703, 2010.

20. Gambacorti-Passerini C, Messa C and Pogliani EM: Crizotinib in anaplastic large-cell lymphoma. N Engl J Med 364: 775-776, 2011
21. Mossé YP, Lim MS, Voss SD, Wilner K, Ruffner K, Laliberte J, Rolland D, Balis FM, Maris JM, Weigel BJ, et al: Safety and activity of crizotinib for paediatric patients with refractory solid tumours or anaplastic large-cell lymphoma: A Children's Oncology Group phase 1 consortium study. Lancet Oncol 14: 472-480, 2013.

22. Butrynski JE, D'Adamo DR, Hornick JL, Dal Cin P, Antonescu CR, Jhanwar SC, Ladanyi M, Capelletti M, Rodig SJ, Ramaiya N, et al: Crizotinib in ALK-rearranged inflammatory myofibroblastic tumor. N Engl J Med 363: 1727-1733, 2010.

23. Wong KM, Noonan S, O'Bryant C and Jimeno A: Alectinib for the treatment of ALK-positive stage IV non-small cell lung cancer. Drugs Today (Barc) 51: 161-170, 2015.

24. Loong HH, Mok K, Leung LK and Mok TS: Crizotinib in the management of advanced-stage non-small-cell lung cancer. Future Oncol 11: 735-745, 2015.

25. Debelenko LV, Raimondi SC, Daw N, Shivakumar BR, Huang D, Nelson M and Bridge JA: Renal cell carcinoma with novel VCL-ALK fusion: New representative of ALK-associated tumor spectrum. Mod Pathol 24: 430-442, 2011.

26. Mariño-Enríquez A, Ou WB, Weldon CB, Fletcher JA and Pérez-Atayde AR: ALK rearrangement in sickle cell trait-associated renal medullary carcinoma. Genes Chromosomes Cancer 50: 146-153, 2011.

27. Smith NE, Deyrup AT, Mariño-Enriquez A, Fletcher JA, Bridge JA, Illei PB, Netto GJ and Argani P: VCL-ALK renal cell carcinoma in children with sickle-cell trait: The eighth sickle-cell nephropathy? Am J Surg Pathol 38: 858-863, 2014

28. Sukov WR, Hodge JC, Lohse CM, Akre MK, Leibovich BC Thompson RH and Cheville JC: ALK alterations in adult renal cell carcinoma: Frequency, clinicopathologic features and outcome in a large series of consecutively treated patients. Mod Pathol 25: 1516-1525, 2012.

29. Hodge JC, Pearce KE and Sukov WR: Distinct ALK-rearranged and VCL-negative papillary renal cell carcinoma variant in two adults without sickle cell trait. Mod Pathol 26: 604-605, 2013.

30. Lee C, Park JW, Suh JH, Nam KH and Moon KC: ALK-positive renal cell carcinoma in a large series of consecutively resected Korean renal cell carcinoma patients. Korean J Pathol 47: 452-457, 2013

31. Xie S, Li Y, Li X, Wang L, Yang N, Wang Y and Wei H: Mer receptor tyrosine kinase is frequently overexpressed in human non-small cell lung cancer, confirming resistance to erlotinib. Oncotarget 6: 9206-9219, 2015.

32. Zhang X, Zhang S, Yang X, Yang J, Zhou Q, Yin L, An S, Lin J, Chen S, Xie Z, et al: Fusion of EML4 and ALK is associated with development of lung adenocarcinomas lacking EGFR and KRAS mutations and is correlated with ALK expression. Mol Cancer 9: 188, 2010.

33. Mino-Kenudson M, Chirieac LR, Law K, Hornick JL, Lindeman N, Mark EJ, Cohen DW, Johnson BE, Jänne PA, Iafrate AJ and Rodig SJ: A novel, highly sensitive antibody allows for the routine detection of ALK-rearranged lung adenocarcinomas by standard immunohistochemistry. Clin Cancer Res 16: 1561-1571, 2010.

34. Roskoski R Jr: Anaplastic lymphoma kinase (ALK): Structure, oncogenic activation, and pharmacological inhibition. Pharmacol Res 68: 68-94, 2013.

35. Debelenko LV: Reply to 'Distinct ALK-rearranged and VCL-negative papillary renal cell carcinoma variants in two adults without sickle cell trait'. Mod Pathol 26: 605-607, 2013.

36. Kuroda N, Sugawara E, Kusano H, Yuba Y, Yorita K and Takeuchi K: A review of ALK-rearranged renal cell carcinomas with a focus on clinical and pathobiological aspects. Pol J Pathol 69: 109-113, 2018.

This work is licensed under a Creative Commons Attribution-NonCommercial-NoDerivatives 4.0 International (CC BY-NC-ND 4.0) License. 